\title{
Anti-CEACAM6 Antibody BAY1834942
}

National Cancer Institute

\section{Source}

National Cancer Institute. Anti-CEACAM6 Antibody BAY1834942. NCI Thesaurus. Code C159609.

A humanized monoclonal antibody directed against the immune checkpoint regulator carcinoembryonic antigen-related cell adhesion molecule 6 (CEACAM6; CEACAM-6; CD66c), with potential immune checkpoint inhibitory and antineoplastic activities. Upon administration, anti-CEACAM6 antibody BAY1834942 targets, binds to and blocks the activity of CEACAM6 expressed on various tumor and immune cells, including T-cells. Blocking CEACAM6 signaling abrogates effector T-cell inhibition, activates antigenspecific T-lymphocytes, increases secretion of T-cell cytokines and effector molecules, and enhances cytotoxic T-cell-mediated tumor cell lysis, which results in a reduction in tumor cell proliferation. CEACAM6, an immune checkpoint receptor, is associated with tumor-mediated immune suppression. Elevated CEACAM6 expression is associated with advanced tumor stages and poor prognosis. 